\title{
Optimal configuration of multiple-focused ultrasound transducers for external hyperthermia
}

\author{
Win-Li Lin \\ Institute of Biomedical Engineering, National Taiwan University, Taipei, Taiwan \\ Yung-Yaw Chen and Shu-Yuh Lin \\ Department of Electrical Engineering, National Taiwan University, Taipei, Taiwan \\ Jia-Yush Yen \\ Department of Mechanical Engineering, National Taiwan University, Taipei, Taiwan \\ Ming-Jium Shieh ${ }^{\text {a) }}$ \\ Institute of Biomedical Engineering, National Taiwan University, Taipei, Taiwan \\ Te-Son Kuo \\ Department of Electrical Engineering, National Taiwan University, Taipei, Taiwan
}

(Received 11 August 1998; accepted for publication 23 June 1999)

\begin{abstract}
External ultrasound hyperthermia is considered to be a very flexible modality for heating deepseated tumors owing to its penetration and focusing ability. However, using this flexibility requires that many complicated, interacting decisions be made to obtain optimal treatment. This paper presents the feasibility of arranging multiple-focused ultrasound transducers to produce an appropriate heating pattern for a specific treatment, based on the optimal scan parameters obtained from an optimization algorithm. The variable scan parameters of the heating system optimized are the transducer tilt and rotation angles, focal depth, scan radius, and output acoustical power. After obtaining the optimal scan parameters, multiple transducers are systematically arranged according to these scan parameters. Three-dimensional ultrasound power deposition and temperature distribution for a specific treatment are calculated for this multiple ultrasound transducer system. A more uniform temperature distribution in the treatment region for a large, highly perfused tumor can be achieved by scanning the system with respect to the central scan axis and/or swinging the transducers inwards and outwards. The maximum heating depth of focused ultrasound transducers used in this heating system is also studied. Simulation results demonstrate that the optimal arrangement of this multiple-focused ultrasound transducer system is highly promising for heating deep, large, and highly perfused tumors. (C) 1999 American Association of Physicists in Medicine.
\end{abstract}

[S0094-2405(99)02109-4]

Key words: external hyperthermia, multiple-focused ultrasound transducer system, optimal configuration, optimization algorithm

\section{INTRODUCTION}

Owing to the physical characteristics of living tissues, ultrasound energy can be delivered to predetermined depths in soft tissues and different power deposition patterns can be produced. ${ }^{1}$ In order to overcome the effect of attenuation, and to deliver more energy into a deep-seated tumor, focused or multiple overlapping fields at a low frequency should be used. When using spherically focused transducers to appropriately deposit the ultrasound energy to cover the entire treatment volume, the focal volume can be scanned mechanically or multiple foci can be formed simultaneously with/ without mechanically scanning. Lele ${ }^{2}$ performed the most extensive studies using mechanical scanning techniques. According to those results, if the ultrasound beam was scanned near the periphery of the tumor, blood flow and thermal conductivity would produce a more homogeneous temperature distribution throughout the entire tumor volume. Hynynen et $a l .^{3}$ expanded the above concept using an ultrasound system adapted from an Octoson diagnostic scanner. That prototype system used four or six therapeutic transducers arranged on a gantry that could scan under computer control with five degrees of freedom. The foci of the four or six beams overlapped in the imaging plane. The improved version of the University of Utah scanned focused ultrasound hyperthermia system ${ }^{4}$ has additional flexibility and automated control features, which should allow a larger variety of tumor sites and larger tumor volumes to be more easily and reliably heated to therapeutic levels. Preliminary experience ${ }^{5}$ was reported for the Sonotherm 6500 scanned focused ultrasound system, which is technically similar to the Arizona SFUS (scanned focused ultrasound system). Further research and development were required to lead to a better realization of the potential of the SFUS technique for tumor treatments. Hunt et al. ${ }^{6}$ used a conically shaped annu- 
lar array transducer to form a line focus and the beam was scanned to cover the treatment volume. Lindsley et al. ${ }^{7}$ studied the temperature distributions and thermal dosimetry of a Helios ultrasound system for deep heating, which uses a rotating annular multiple transducer array consisting of 30 transducers mounted on a spherical shell and arranged in four concentric rings. During treatment, the array can be slowly rotated to smooth spatially the power deposition pattern to achieve a more uniform heating. Lin et al. ${ }^{8}$ examined the optimal frequency and configured the ultrasound energy deposition schema for various sizes and locations of breast tissues when a portion or the entire cylindrical ultrasound transducer is employed for breast hyperthermia treatment. Their results demonstrated that the breast size, the ultrasound frequency, the shifting distance between the central axes of the breast and the transducer, and the active portion of the cylindrical transducer are the potential parameters for influencing the distribution of the specific absorption rate (SAR) ratio. Svensson et al. ${ }^{9}$ assessed a focused ultrasound hyperthermia system consisting of $56,3 \mathrm{~cm}^{2}$, individually controlled transducer elements mounted on a spherical shell (24 $\mathrm{cm}$ radius) having a $30 \mathrm{~cm}$ aperture. Nussbaum et al. ${ }^{10}$ evaluated a 30-beam ultrasonic hyperthermia system developed by Varian Associates ${ }^{11}$ in a large, musclelike phantom and in live, adult pigs. Their results demonstrated the potential of the system for producing localized and adjustable heating patterns over clinically useful volumes. Kirkhorn et $a .^{12}$ designed high-energy ultrasound equipment with treatment heads consisting of six or seven focused ultrasound transducers. Their results showed that well-defined pressure foci could be produced at the geometrical foci of the transducer unit. The studies ${ }^{13,14}$ of a spherical-section ultrasound phased array applicator showed that two-dimensional large aperture phased arrays with an optimal heating strategy (mainly delivering peak power values around the periphery of the tumor volume) could provide precise control over the heating pattern in three dimensions for deep localized hyperthermia. However, with the increase in the number of elements, both the control and fabrication process involved greater complexity. Moros et al. ${ }^{15,16}$ developed a scanning ultrasound reflector linear array system for the simultaneous delivery of hyperthermia and ionizing radiation to increase thermal radiosensitization in clinical treatment. They investigated the conformability of power deposition and control of penetration depth for this multielement ultrasound heating system.

During scanned focused ultrasound hyperthermia, the focus is moved at a selected speed over a chosen path repeatedly to produce a therapeutic temperature elevation in the target volume. Thus, the energy input into a tissue volume within the scanning path is periodic. A sharp temperature increase occurs when the ultrasound beam is present at a location and a subsequent temperature decay occurs while other parts of the target volume are scanned. The magnitude of this temperature variation depends on the scan period (the time that the ultrasound beam takes to complete the entire scan path) and the blood perfusion rate for a given target temperature. A longer scan period and a higher perfusion rate will result in a higher temperature variation for a given transducer. The results of Moros et al. ${ }^{17}$ and Lele ${ }^{18}$ suggested that scans using sharply focused transducers should be completed within 3-10 s in order to avoid significant temperature variations (the magnitude is about $0.7-2.3{ }^{\circ} \mathrm{C}$ for a blood perfusion of $\left.10 \mathrm{~kg} \mathrm{~m}^{-3} \mathrm{~s}^{-1}\right) .{ }^{17}$ To heat a large, highly perfused tumor, multiple scans with proper spacing between scan paths is required (less than or equal to the diameter of the beam focus ${ }^{17}$ ). To avoid overheating caused by the overlapping of the focused beams (mainly in the region in front of the target volume), tilt and rotation of the transducer (Lele, ${ }^{18}$ Lin et al. ${ }^{19}$ ) can effectively prevent such overheating problem. This problem can also be reduced by using multiple beams and switching off each beam when it passes through the central part of the scan circle to the opposite side. ${ }^{20}$ When the transducer is tilted and rotated, the circle over which the physical transducer scans increases significantly (see Fig. 1). A much higher scanning speed for the transducer is needed to complete the entire scan path within 3-10 $\mathrm{s}$ when a multiple-circle scan is used for heating a large tumor. However, the scanning speed of the transducer is constrained by the water resistance and turbulence if no essential provisions are made to avoid this. ${ }^{1}$ In addition, the greater intensity of a single scanning focal zone transducer may cause the patient discomfort ${ }^{21}$ and the induction of transient cavitation. $^{22}$

This paper describes the investigation of the optimal configuration of a multiple-focused ultrasound transducer system. A two-dimensional optimization algorithm ${ }^{19}$ based on a temperature objective function is first employed to search for the optimal scan parameters. Next, multiple transducers are arranged with these optimal scan parameters to form a heating configuration to approximate the two-dimensional optimization result. This optimal configuration of multiple transducers can deliver suitable power to produce an appropriate temperature distribution for a given treatment. To overcome the effect of high blood perfusion on the temperature distribution, rotating the system with respect to the central scan axis and/or swinging the transducers inwards and outwards can be employed to yield a more uniform temperature distribution within the treatment region. The selection and limitations of spherically focused ultrasound transducers is also presented.

\section{METHOD}

\section{A. Ultrasound power deposition}

Figure 1 depicts the geometry of an ultrasound heating system with multiple-focused transducers arranged below the treatment volume and the ultrasound power is emitted from the transducers. Tilt angle $(\theta)$, rotation angle $(\phi)$, focal depth $\left(z_{s}\right)$, and scan radius $\left(r_{s}\right)$ are the four scan parameters which can be varied by the operator to obtain the desired heating configuration; the magnitude of ultrasound output power can also be varied. $R_{s}$ is the scan radius on the transducer plane and $r_{w}$ is the radius of the acoustical window on the skin for the entire ultrasound beam. When the transducers are scanning sufficiently fast, the power deposition patterns can be 


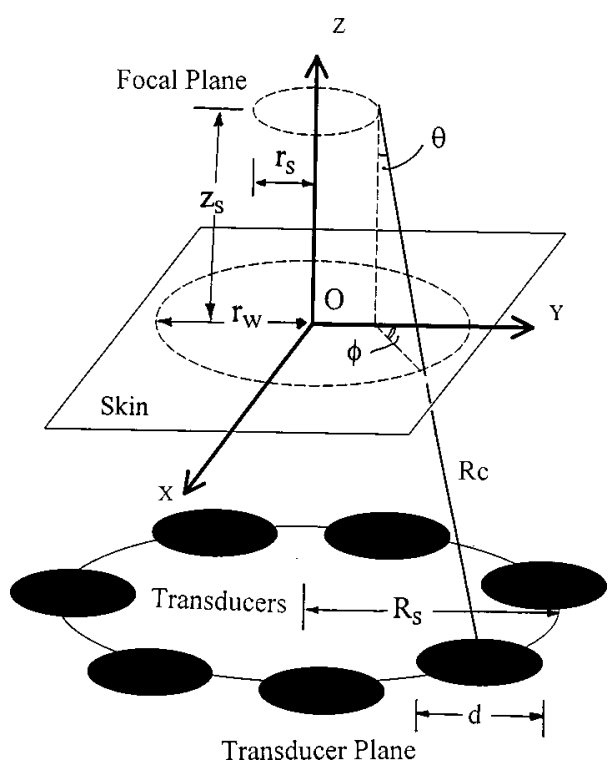

FIG. 1. Schematic diagram of the geometry studied. Transducer's tilt angle $(\theta)$ and rotation angle $(\phi)$, scan radius $\left(r_{s}\right)$, and focal depth $\left(z_{s}\right)$ are the scan parameters which can be varied to produce an optimal power deposition for the treatment. And the output power from the transducer is also varied. Focal depth is the distance between the focal plane and the skin, which is located in the $x-y$ plane at $z=0$. The transducers are located below the skin plane.

considered to be symmetric about the $z$ axis (central scan axis) for tissues with homogeneous ultrasound absorption properties. To calculate the absorbed power deposition, the program developed by Swindell et al. ${ }^{23}$ was used to simulate the ultrasound power deposition for a stationary transducer whose axis is normal to the skin. This program solves the Rayleigh-Sommerfeld diffraction integral and exponentially attenuates the power along the propagation direction for a given frequency-dependent attenuation coefficient. Generally, in the simulations of ultrasound hyperthermia, all of the attenuated energy has been assumed to be absorbed locally. The scattered energy will be absorbed, and during ultrasound hyperthermia most of the scattered waves are absorbed in the treatment volume. ${ }^{1}$ Hence, scattering effects were neglected and all incident energy was assumed to be absorbed by the tissue in this study. To tilt and rotate the stationary power deposition through given angles, the programs developed by Moros $^{24}$ and by Lin et al. ${ }^{19}$ were employed. To develop a power deposition pattern for a given scan radius, the power field was translated away from the central scan axis a distance equal to the scan radius.

The two-dimensional optimization algorithm ${ }^{19}$ was used to determine the transducer's scan parameters for single or multiple-circle scans which can produce an appropriate power deposition to meet the requirements for a specific treatment. Multiple-stationary transducers with these optimal scan parameters were uniformly arranged to divide equally the scan circle to form a heating configuration. The maximum number of transducers allowable is determined by the transducer diameter and the available space on the transducers' plane that can be determined by the transducer diameter, radius of curvature, tilt and rotation angles, and scan radius.

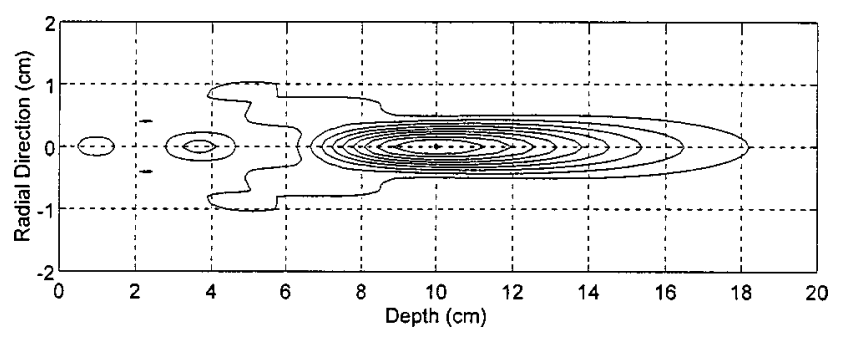

FIG. 2. Stationary normalized $(0-100 \%)$ power intensity distribution for the transducer used in this study which has a $10 \mathrm{~cm}$ diam, a $20 \mathrm{~cm}$ curvature radius, and a $0.5 \mathrm{MHz}$ frequency. The outer curve shows the $10 \%$ contour; other curves are at $10 \%$ intervals (attenuation $10 \mathrm{~Np} \mathrm{~m}^{-1} \mathrm{MHz}^{-1}$ ).

To calculate the three-dimensional (3D) power deposition formed by these multiple-stationary transducers, the ultrasound power deposition for a single transducer with optimal scan parameters was first calculated using the above procedure. Then the power deposition of this single transducer was rotated with respect to the central scan axis with an appropriate angle to develop the power deposition for individual transducer located at different orientation. Finally, the power depositions of all the transducers were summed to form the entire 3D power deposition produced by the multiple-focused ultrasound transducers with this configuration. Thus, the appropriate 3D power deposition formed by multiple-focused ultrasound transducers for a specific treatment was obtained. An ultrasound transducer with a diameter of $10 \mathrm{~cm}$, a curvature radius of $20 \mathrm{~cm}$, and a frequency of 0.5 $\mathrm{MHz}$ was chosen for this study. Figure 2 presents the stationary, normalized $(0-100 \%)$ attenuated power deposition of this transducer. The ultrasound attenuation is $4.0-29,5.0-$ $9.0,3.0-10,3.2-18,4.4-15 \mathrm{~Np} \mathrm{~m}^{-1}$ for brain, fat, kidney, liver, and muscle, respectively, ${ }^{25-28}$ at a temperature of $37^{\circ} \mathrm{C}$ and a frequency of $1 \mathrm{MHz}$. To obtain a simple but realistic approximation of the power deposition, an average value of $10 \mathrm{~Np} \mathrm{~m}^{-1} \mathrm{MHz}^{-1}$ for muscle has been considered during this simulation study. Moreover, the power generated from all parts of the transducer was assumed to be attenuated equally at each depth. ${ }^{19,24}$

\section{B. Temperature solver}

Pennes ${ }^{29}$ steady-state bio-heat transfer equation (BHTE) was used to solve for the temperature distribution associated with a given power deposition,

$$
-k \nabla^{2} T+W c_{b}\left(T-T_{\mathrm{ar}}\right)=Q .
$$

The thermal conductivity $(k)$ was $0.5\left(\mathrm{~W} \mathrm{~m}^{-1}{ }^{\circ} \mathrm{C}^{-1}\right)$, the specific heat of blood $\left(c_{b}\right)$ was $3770\left(\mathrm{~J} \mathrm{~kg}^{-1}{ }^{\circ} \mathrm{C}^{-1}\right)$, and the arterial temperature $\left(T_{\text {ar }}\right)$ was $37^{\circ} \mathrm{C}$. The density of absorbed ultrasonic power $(Q)$ was obtained using the above procedure for a given configuration of multiple-focused ultrasound transducers. A uniform blood perfusion $(W)$ was used and metabolism was neglected owing to its small contribution to the temperature change. ${ }^{30,31}$ Other physiological properties might change and affect the temperature distribution during the hyperthermia treatment. ${ }^{25,28,32,33}$ However, a good approximation can be obtained when the physiological proper- 
ties (except the blood perfusion) are assumed to remain constant throughout the entire field and treatment duration within the temperature range used in hyperthermia. The 3D steady-state BHTE was numerically approximated using successive-over-relaxation and the method of central finite difference. ${ }^{34,35}$ The radius and the thickness of the cylindrical tissue volume studied were 10 and $20 \mathrm{~cm}$, respectively. The boundary condition for the top, bottom, and outer surface was a constant temperature of $37^{\circ} \mathrm{C}$. The above BHTE is a simplification that neglects the effects of discrete blood vessels and the redistribution of thermal energy within the local vascular network. However, this BHTE offers a practical approach for modeling biothermal processes ${ }^{31,36,37}$ and performing general parameter studies.

\section{RESULTS}

\section{A. Single circle scan}

The center of a small tumor, with a $3 \mathrm{~cm}$ diam by $3 \mathrm{~cm}$ long cylindrical segment, is located at a depth of $10 \mathrm{~cm}$. A uniform blood perfusion with $5 \mathrm{~kg} \mathrm{~m}^{-3} \mathrm{~s}^{-1}$ was assumed for both normal tissue and tumor tissue. Using the twodimensional (2D) optimization search, ${ }^{19}$ the optimal values for tilt angle $(\theta)$, rotation angle $(\phi)$, scan radius $\left(r_{s}\right)$, and focal depth $\left(z_{s}\right)$ were determined to be $40^{\circ}, 90^{\circ}, 1.0 \mathrm{~cm}$, and $10 \mathrm{~cm}$, respectively. Figures 3(a) and 3(b) depict the 2D ultrasound power deposition and temperature distribution formed by this set of scan parameters. The $43^{\circ} \mathrm{C}$ isothermal line is located along the tumor boundary, and the highest temperature within the tumor is approximately $48^{\circ} \mathrm{C}$. For the configuration of multiple-stationary transducers, seven ultrasound transducers with the above scan parameters were arranged uniformly to divide equally the scan circle to approximate this single circle scan. Figures 3(c) and 3(d), the 2D temperature distributions on the $x-z$ and $y-z$ planes, show that the temperature contour plots are close to the $2 \mathrm{D}$ optimization result [Fig. 3(b)]. Figure 3(e) is the six 2D temperature surface plots of the horizontal planes located at depths of 8.4, 9.3, 10.2, 11.1, 12.0, and $12.9 \mathrm{~cm}$, respectively. Seven temperature peaks, as produced by these stationary focused ultrasound transducers, can clearly be viewed on the top and bottom plots.

\section{B. Multiple circle scans}

For larger tumors, multiple-circle scans become necessary to produce an appropriate temperature distribution. Doublecircle scans were employed to treat a tumor with $5 \mathrm{~cm}$ diameter by $5 \mathrm{~cm}$ thickness and located at a depth of $10 \mathrm{~cm}$. A uniform blood perfusion with $5 \mathrm{~kg} \mathrm{~m}^{-3} \mathrm{~s}^{-1}$ was assumed for both normal tissue and the tumor region. Through the 2D optimization search, $\theta, \phi, r_{s}$, and $z_{s}$ were determined to be $20^{\circ}, 90^{\circ}, 1 \mathrm{~cm}$, and $10 \mathrm{~cm}$ for the inner circle scan and $40^{\circ}$, $90^{\circ}, 2 \mathrm{~cm}$, and $10 \mathrm{~cm}$ for the outer circle scan. Figures $4(\mathrm{a})$ and 4(b) present the 2D ultrasound power deposition and temperature distribution formed by these double-circle scans. For the arrangement of multiple-stationary transducers, four ultrasound transducers with the inner scan parameters were used for the inner scan circle, and seven transducers with the outer scan parameters for the outer scan circle. Figures 4(c)4(e) plot the 2D temperature distributions on different planes for the 3D temperature distribution produced by these eleven stationary transducers. Figures 4(c) and 4(d) are the temperature contour plots on the $x-z$ and $y-z$ planes, while Fig. 4(e) shows the six 2D temperature surface plots for the horizontal planes located at depths of 7.2, 8.4, 9.6, 10.8, 12, and $13.2 \mathrm{~cm}$, respectively. On the top plots of Fig. 4(e), eleven temperature peaks, resulting from these eleven stationary focused ultrasound transducers, can be viewed.

As either blood perfusion or the gap between ultrasound beams increases, the smoothing effect of bioheat transfer may not be sufficient to yield an appropriately uniform temperature distribution within the treatment region. To obtain a more uniform power deposition along the scan circles, this multiple-transducer system can be rotated with respect to the central scan axis. There are multiple-focal zones formed by the focused ultrasound transducers on each scan circle. Hence the ultrasound intensity on an individual focal zone of this multiple-transducer system is much smaller than that of a single overlapping focal zone formed by scanned multiplefocused transducers ${ }^{3}$ or than that of a single focal zone formed by a single scanned focused transducer., ${ }^{2,4}$ As a result, the scanning speed of this multiple-transducer system does not need to be as great and the temperature fluctuation, especially on the scan circles, caused by the system scanning is much smaller. ${ }^{24}$ The ultimate temperature distribution for scanning this multiple-transducer system is the $2 \mathrm{D}$ convolution result, as shown in the previous $2 \mathrm{D}$ optimization study [Figs. 3(b) and 4(b)].

Due to the geometrical constraints shown in Fig. 1 (transducer diameter, curvature radius, tumor size and depth, etc.), the scan circles may not be able to be arranged as close as the treatment required for this multiple-transducer system. The nonuniform temperature distribution between the scan circles may become undesirable due to high blood perfusion and the gap between the scan circles. To solve this problem, the scan circles can swing inward to and outward from the central scan axis. Figures 5(a)-5(c) are the temperature distributions for a blood perfusion of $10 \mathrm{~kg} \mathrm{~m}^{-3} \mathrm{~s}^{-1}$ when scan radius is equal to $0.5,1.0$, and $1.5 \mathrm{~cm}$, respectively, while the other scan parameters are maintained the same as those in Fig. 3. These temperatures show that the central region with low temperature is getting larger as the scan radius increases. Figure 5(d) is the temperature distribution produced by the focal zone of a transducer swinging back and forth from 0.5 to $1.5 \mathrm{~cm}$ with a power weighting $1: 1: 3$. This temperature indicates that the low temperature in the central region disappears and a large, uniform heating region can be obtained. This swinging redistributes the ultrasound power to provide a more uniform deposition in the radial direction and hence a more uniform temperature distribution in the treatment region can be obtained.

\section{DISCUSSION}

The arrangement geometry of the multiple-focused transducer system shown in Fig. 1 indicates that the maximum 

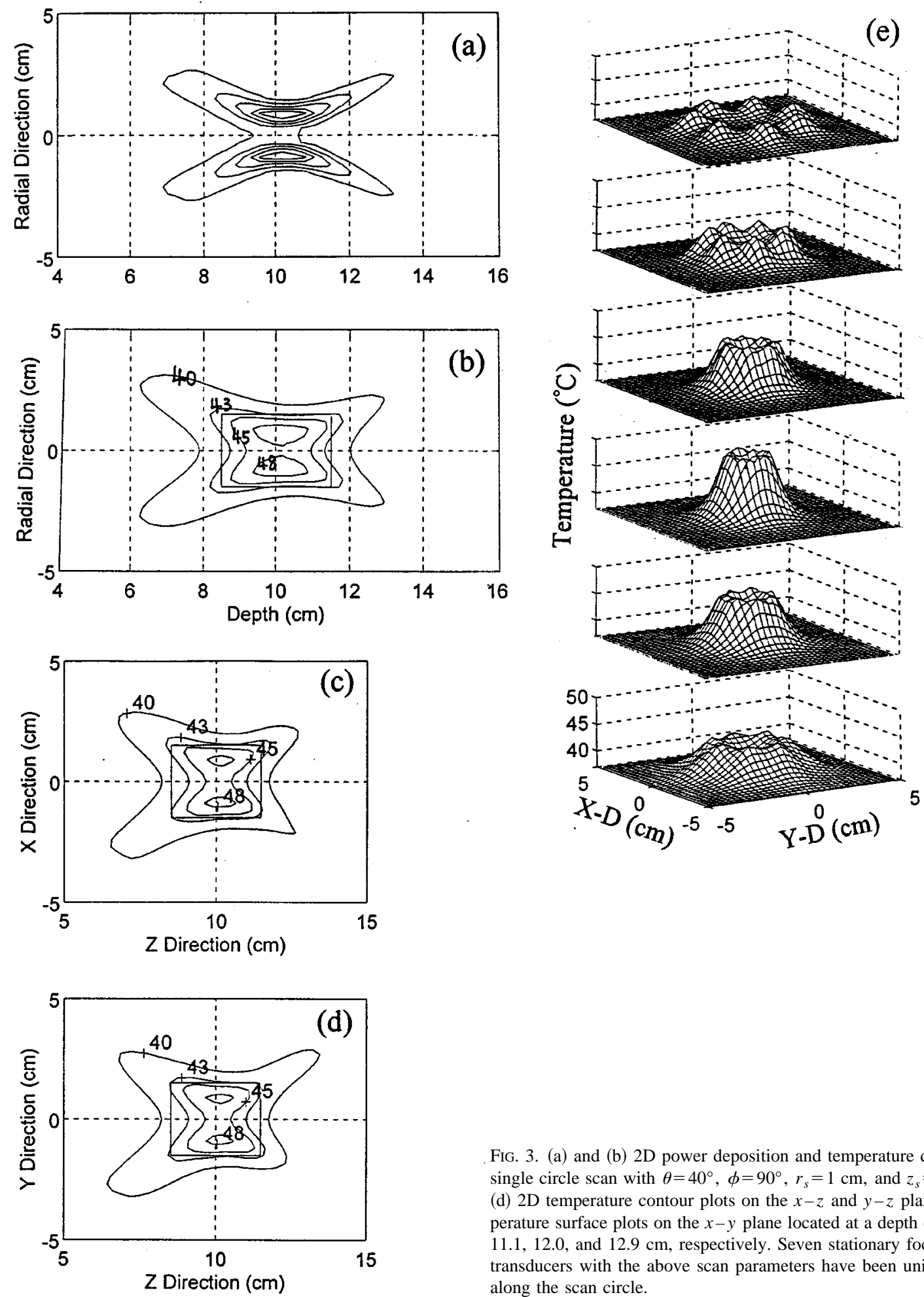

number of transducers allowed to be arranged along a scan circle can be determined from the relationship between $\theta, \phi$, $R_{s}, r_{s}$, transducer diameter $(d)$, and curvature radius $\left(R_{c}\right)$ by

number of transducers allowable

$$
=\max \text { integer of }\left(2 \pi R_{s} / d\right),
$$

where

$$
R_{s}^{2}=r_{s}^{2}+\left(R_{c} \sin \theta\right)^{2}-2 r_{s} R_{c} \sin \theta \cos (180-\phi) .
$$

For a stationary multiple-transducer system, the transducers arranged along each scan circle should be as great as possible to reduce the temperature difference on the scan circle, especially for a high perfusion case.

The evaluation of the effects of power penetration and acoustical attenuation on the optimal temperature distribu- 

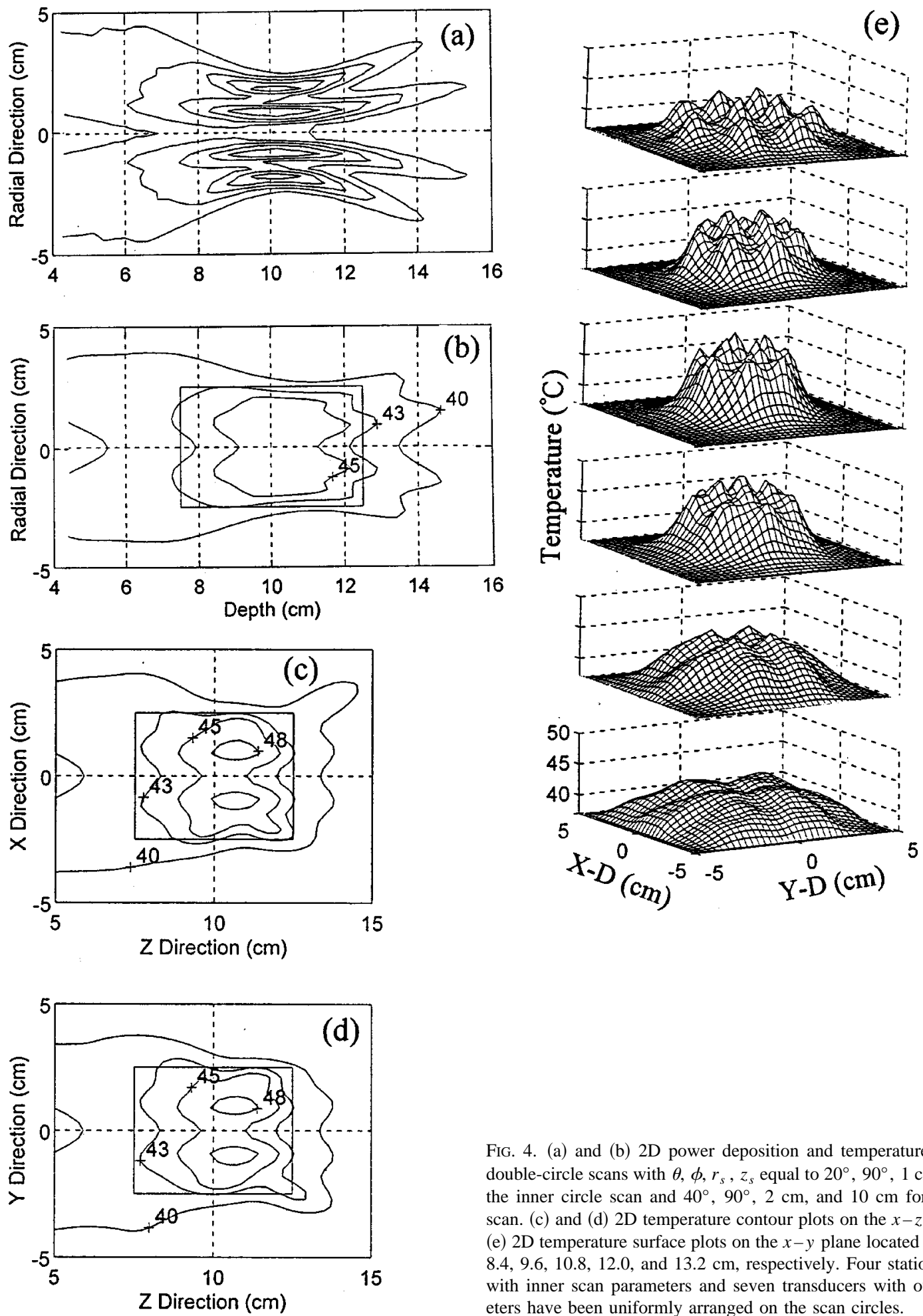

tions and scan parameters has been done for the 2D optimization study. ${ }^{19}$ Those results revealed that generally there were no significant differences in either optimal temperature distributions or optimal scan parameters $\left(\theta, \phi\right.$, and $\left.r_{s}\right)$ when the tumor's center was set at depths from 2 to $7 \mathrm{~cm}$ below the skin for a $2 \mathrm{~cm} \times 2 \mathrm{~cm}$ tumor with a constant blood perfusion. The optimal focal depth $\left(z_{s}\right)$ was coupled with tumor depth and the applied power increased with tumor depth due

FIG. 4. (a) and (b) 2D power deposition and temperature distribution for double-circle scans with $\theta, \phi, r_{s}, z_{s}$ equal to $20^{\circ}, 90^{\circ}, 1 \mathrm{~cm}$, and $10 \mathrm{~cm}$ for the inner circle scan and $40^{\circ}, 90^{\circ}, 2 \mathrm{~cm}$, and $10 \mathrm{~cm}$ for the outer circle scan. (c) and (d) 2D temperature contour plots on the $x-z$ and $y-z$ planes. (e) 2D temperature surface plots on the $x-y$ plane located at a depth of 7.2, 8.4, 9.6, 10.8, 12.0, and $13.2 \mathrm{~cm}$, respectively. Four stationary transducers with inner scan parameters and seven transducers with outer scan parameters have been uniformly arranged on the scan circles.

to the ultrasound attenuation in tissue. Those previous results demonstrated that the heating pattern could be used for tumors located at different depths as long as the tumor size and shape were maintained. When this multiple-focused ultrasound transducer system with/without mechanical scanning is employed for hyperthermia treatments, the factors determining the treatable tumor depth and size are the transducer characteristics used (diameter, frequency, radius of curva- 

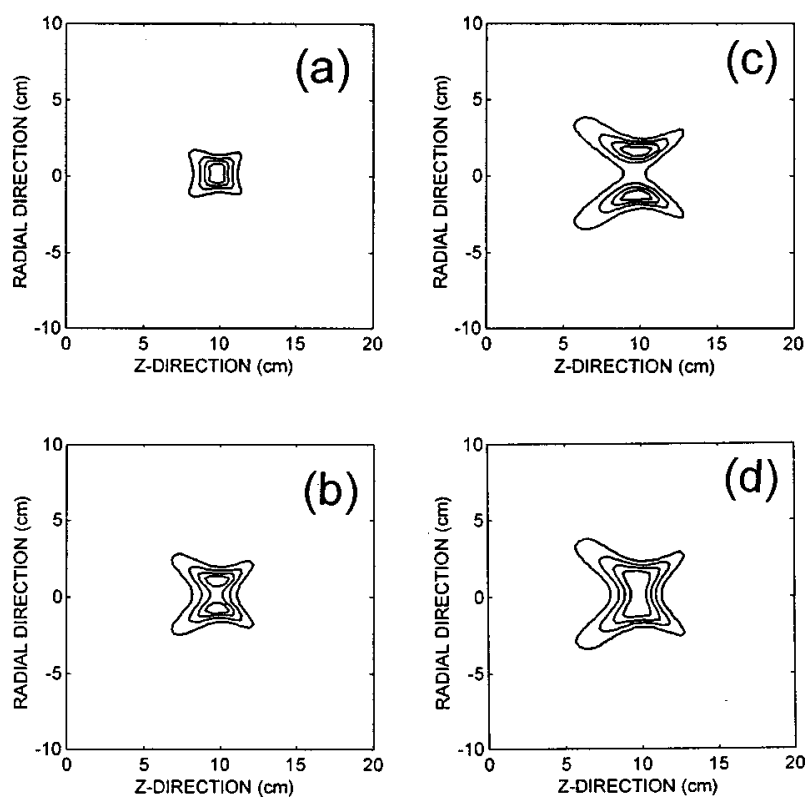

FIG. 5. (a) - (c) 2D temperature distributions for a single circle scan with $\theta$ $=40^{\circ}, \phi=90^{\circ}, z_{s}=10 \mathrm{~cm}$, while $r_{s}$ is equal to $0.5,1.0$, and $1.5 \mathrm{~cm}$, respectively. (d) 2D temperature distribution for the focal zone of transducer swinging inward and outward the central scan axis from $r_{s}=0.5$ to $1.5 \mathrm{~cm}$. A blood perfusion of $5 \mathrm{~kg} \mathrm{~m}^{-3} \mathrm{~s}^{-1}$ is used.

ture) and the power density gain at the tumor depth. The power density gain at the tumor depth is related to ultrasound attenuation and the geometric gain of the system arrangement. The geometric gain of the entire ultrasound beam can be defined as the ratio of the acoustical window radius to tumor size $\left(r_{w}^{2} / r_{t}^{2}\right.$, where $r_{w}$ denotes the intersection radius of the skin and the entire ultrasound beam formed by this system; $r_{t}$ represents the tumor radius). The radius of acoustical window $\left(r_{w}\right)$ can be induced from the configuration of Fig. 1 and given by the following equation when the entire ultrasound beam is modeled as a simple cone:

$$
\begin{aligned}
r_{w}^{2}= & r_{s}^{2}+z_{s}^{2} \tan \left[\theta+\tan ^{-1}(1 / 2 F)\right] \\
& -2 r_{s} z_{s} \tan \left[\theta+\tan ^{1}(1 / 2 F)\right] \cos (180-\phi),
\end{aligned}
$$

where $F$ denotes the transducer $f$ number, defined as the curvature radius of the transducer divided by the transducer diameter.

In order to obtain a power density gain at the tumor depth sufficiently high to produce an appropriate temperature distribution for a given treatment, the geometric gain must be large enough to overcome the acoustical attenuation and heat transfer. The ratio of power density gain for an appropriate heating temperature distribution ranges from 1.8 to 4.5 , depending on tumor depth, tumor size, blood perfusion, ultrasound frequency, and acoustic attenuation. ${ }^{38}$ Hence, the arrangement of this heating system must meet this requirement during treatment of a large and deep tumor when the acoustical window obtained from the optimization algorithm is too large for the practical treatment.
The proposed heating system is formed with multiplefocused ultrasound transducers. In addition to the power density gain at tumor depth produced by the entire ultrasound beam, the transducer characteristics used are also a major factor affecting the treatable tumor depth and size. Figure 6(a) shows the absorbed intensity distribution along the central axis in tissue for a stationary focused ultrasound transducer (a diameter of $10 \mathrm{~cm}$, and a frequency of $1 \mathrm{MHz}$ ) with different radii of curvature. By increasing the curvature radius, the location of focal zone (maximum absorbed intensity) can be pushed deeper into the tissue, the focal region becomes longer, and the relative absorbed intensity gets smaller due to the reduction of transducer focusing ability and the acoustical attenuation in tissue. Figure 6(b) shows the temperature distributions along the central axis corresponding to different distributions of absorbed intensity. The maximum temperature in tissue is set at $50{ }^{\circ} \mathrm{C}$, while the temperature of surface cooling water is $37^{\circ} \mathrm{C}$ and blood perfusion is $5 \mathrm{~kg} \mathrm{~m}^{-3} \mathrm{~s}^{-1}$. Figure $6(\mathrm{~b})$ indicates that the location of the $50{ }^{\circ} \mathrm{C}$ surface moves deeper with the focal zone and the temperature close to the skin rises as the curvature radius increases. At a certain radius of curvature, the tissue temperature close to the skin reaches $45^{\circ} \mathrm{C}$. As the curvature radius increases further, the temperature close to the skin becomes $50{ }^{\circ} \mathrm{C}$ (the maximum heating temperature shifts from the focal zone to the region close to the skin) even though the relative absorbed intensity in the focal zone is still the highest within the entire domain. This indicates that the location of the highest tissue temperature is determined not only by the relative value of the absorbed intensity but also by the effect of bio-heat transfer. The maximum heating depth (MHD) of a stationary focused ultrasound transducer is defined as the distance between the skin and the achievable deepest location of $50{ }^{\circ} \mathrm{C}$ in the depth direction. Comparing the maximum depth of the acoustical focus ${ }^{39}$ and the MHD in Figs. 6(a) and 6(b) indicates that the MHD is shorter than the maximum depth of acoustical focus.

A comprehensive study of MHD has been done for ultrasound transducers with diameters from 3 to $10 \mathrm{~cm}$ and frequencies from 0.5 to $2.0 \mathrm{MHz}$. The result shown in Fig. 7 indicates that the MHD increases with the transducer's diameter but decreases with frequency. A larger transducer with lower frequency provides a deeper MHD. This indicates that a large transducer with low frequencies should be employed for heating deep-seated tumors. This plot can be used as a guideline to select a spherically focused ultrasound transducer with appropriate characteristics for a desired heating depth of hyperthermia treatment. As is known, blood perfusion plays an important role for the temperature distribution in tissue, hence it is necessary to study the effect of perfusion on the MHD. Figure 8 presents this relationship for a $5 \mathrm{~cm}$ diam transducer with different frequencies, showing that a higher perfusion obviously results in a deeper MHD. As the perfusion becomes greater, the pattern of temperature distribution gets closer to that of absorbed ultrasound intensity deposition and MHD approaches the maximum depth of acoustical focus. This means that the maximum depth of 

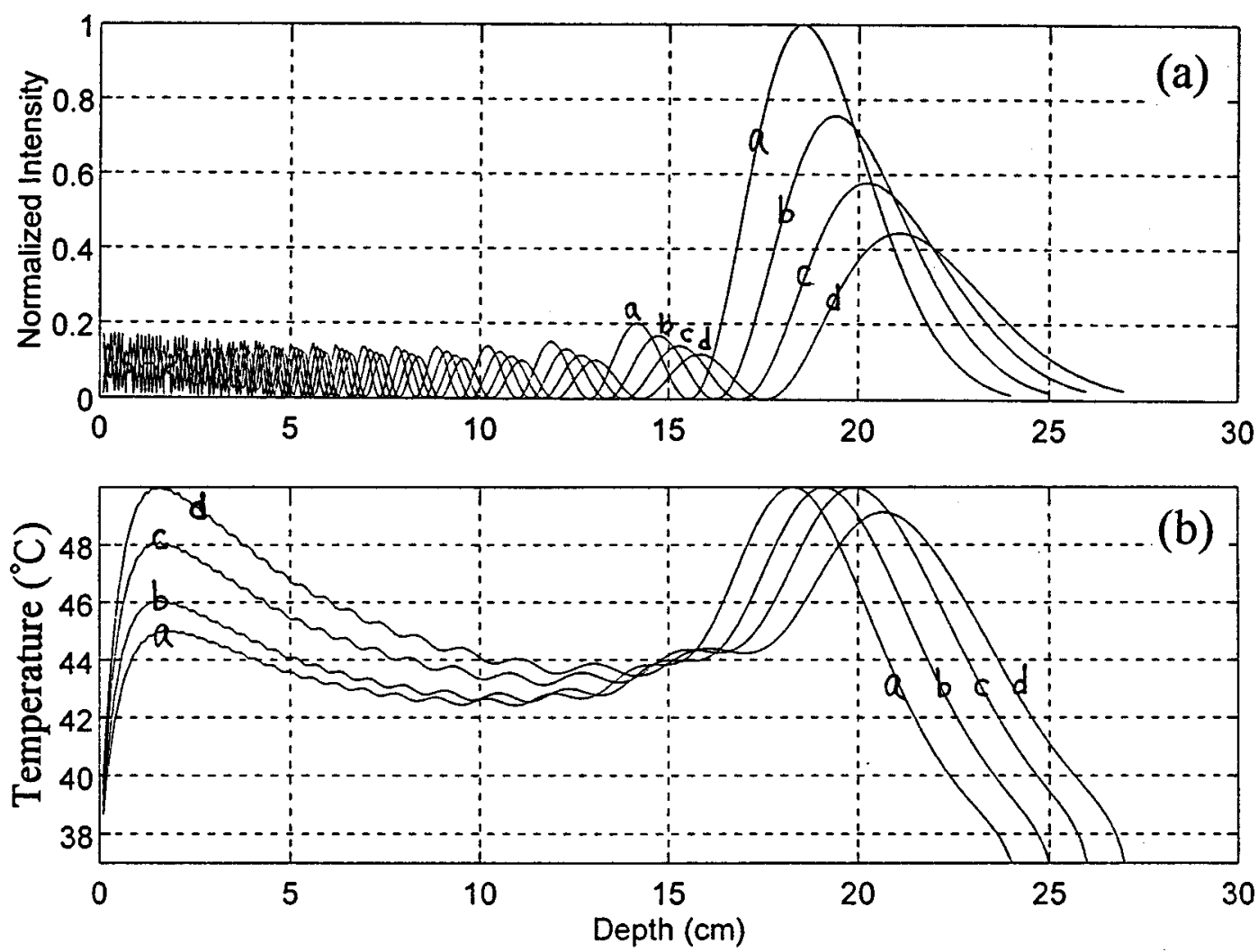

FIG. 6. (a) Absorbed ultrasound intensity distribution; and (b) temperature distribution along the central axis for a stationary focused ultrasound transducer (a diameter of $10 \mathrm{~cm}$, a frequency of $1 \mathrm{MHz}$ ) with various values of curvature radius calculated in tissue (an attenuation coefficient of $10 \mathrm{~Np} \mathrm{~m}^{-1} \mathrm{MHz}^{-1}$, a blood perfusion of $5 \mathrm{~kg} \mathrm{~m}^{-3} \mathrm{~s}^{-1}$, and a surface cooling water temperature of $37^{\circ} \mathrm{C}$ ).

acoustical focus is the maximum value of MHD for a stationary focused ultrasound transducer.

The two-dimensional optimization algorithm ${ }^{19}$ based on the temperature distribution shows how to determine the optimal scan parameters for the transducer(s) which can produce an appropriate power deposition to meet the requirements for a given set of treatment conditions. To realize these optimization results, one can employ a single focused transducer scanned with or multiple-focused transducers arranged according to this set of scan parameters. When a single scanning transducer method is employed, one must monitor the temperature fluctuation on the scan circle path due to the periodic heating and the effect of water resistance on the scanning transducer in water. The temperature fluctuation and the water resistance become serious when multiple-circle scans are used for a scanning focused transducer to treat a large and high perfusion tumor. On the other hand, the arrangement of multiple-focused transducers can effectively reduce the temperature fluctuation due to a much lower intensity at the individual focal zone and a much shorter heating period. The water resistance to the movement of the transducers can also be effectively reduced.

The study of the conformability ${ }^{31,40}$ of a scanned focused ultrasound hyperthermia system had been done for cases where both the tumor diameter and thickness varied from 1 to $3 \mathrm{~cm}$ with a single-circle scan, and from 3 to $5 \mathrm{~cm}$ with a double-circle scan ${ }^{19}$ showing that the optimal scan parameters for the heating system gave good results in that the $43^{\circ} \mathrm{C}$ line could be placed close to the tumor boundary for all conditions. This indicates that the proposed multiplefocused ultrasound transducer system can be arranged with appropriate configurations to produce suitable temperature distributions for tumors with different cylindrical segments, and it is necessary for future studies using the combination of different cylindrical segments to conform the real tumor geometry.

\section{CONCLUSION}

The results of this study demonstrate that an appropriate temperature distribution for the treatment can be achieved by employing an optimization algorithm ${ }^{19}$ to search for the optimal scan parameters and then arranging multiple-focused transducers on an individual scan circle with these scan parameters. Bio-heat transfer can smooth out the temperature nonuniformity between the ultrasound beams for low blood perfusion cases. For a large gap and/or high blood perfusion in which bio-heat transfer cannot smooth out the temperature distribution, rotating the system with respect to the central scan axis and/or swinging the scan circles inwards and outwards can yield a more uniform temperature distribution. This system can be employed to heat a large, deep tumor as long as the geometric gain at the tumor depth can overcome the acoustical attenuation and an appropriate power deposition pattern can be produced. In addition, the result for the MHD of spherically focused ultrasound transducers offers 


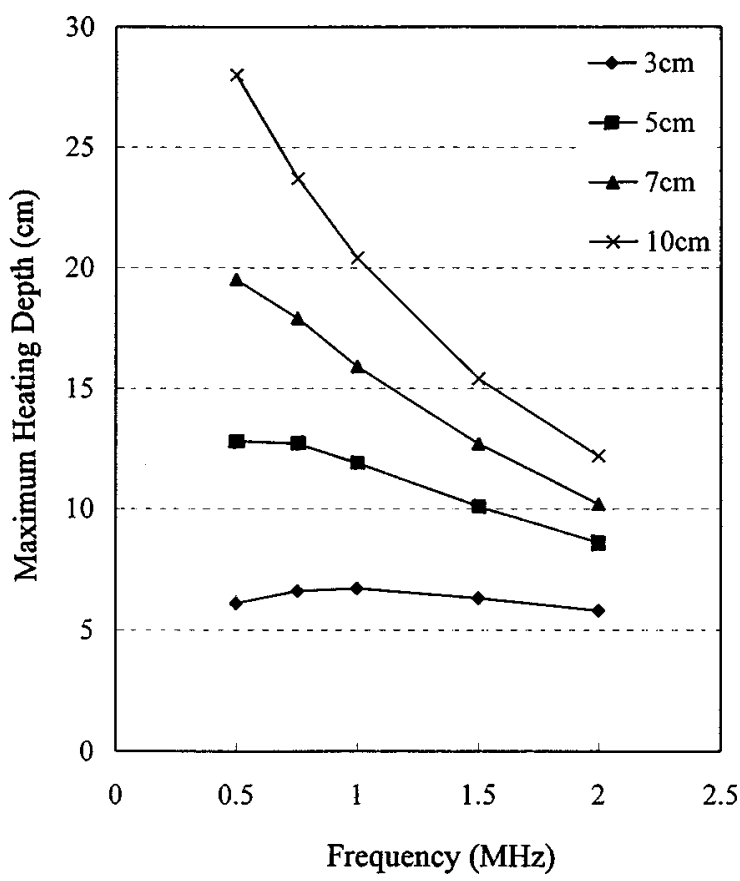

FIG. 7. Maximum heating depth (MHD) for stationary focused ultrasound transducer as a function of frequency and transducer diameter in tissue (an attenuation coefficient of $10 \mathrm{~Np} \mathrm{~m}^{-1} \mathrm{MHz}^{-1}$, a blood perfusion of $5 \mathrm{~kg} \mathrm{~m}^{-3} \mathrm{~s}^{-1}$, and a surface cooling water temperature of $37^{\circ} \mathrm{C}$ ).

the information to select proper characteristics of transducers for a given treatment. The above findings indicate that this multiple-focused ultrasound transducer system is highly promising for hyperthermia of deep, large, and highly perfused tumors.

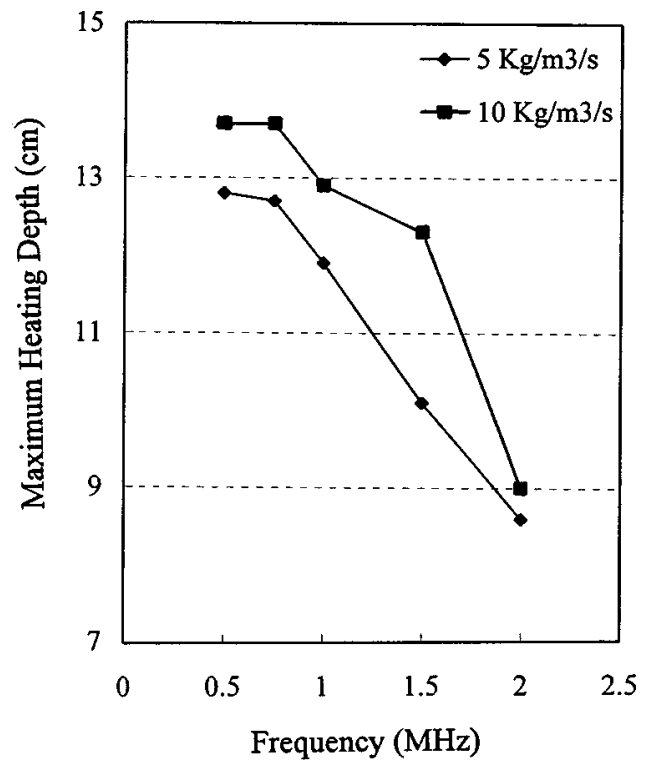

FIG. 8. Maximum heating depth (MHD) for a stationary focused ultrasound transducer as a function of frequency and perfusion in tissue (a transducer diameter of $5 \mathrm{~cm}$, an attenuation coefficient of $10 \mathrm{~Np} \mathrm{~m}^{-1} \mathrm{MHz}^{-1}$, and a surface cooling water temperature of $37^{\circ} \mathrm{C}$ ).

\section{ACKNOWLEDGMENTS}

This work was supported in part by grants from the National Science Council No. (NSC 87-2213-E-002-077), and from the Department of Health No. (DOH 87-HR-635).

${ }^{a}$ Author to whom all correspondence should be addressed.

${ }^{1} \mathrm{~K}$. Hynynen, "Biophysics and technology of ultrasound hyperthermia," in Methods of External Hyperthermic Heating, edited by M. Gautherie (Springer, Berlin, 1990), pp. 61-116.

${ }^{2} \mathrm{P}$. P. Lele, "Ultrasound: Is it the modality of choice for controlled, localized heating of deep tumors?" in Hyperthermia Oncology, edited by J. Overgaard (Taylor and Francis, London, 1984), Vol. 2, pp. 129-154.

${ }^{3}$ K. Hynynen, R. B. Roemer, D. Anhalt, C. Johnson, Z. X. Xu, W. Swindell, and T. Cetas, "A scanned focused multiple transducer ultrasonic system for localized hyperthermia treatments," Int. J. Hyperthermia 3, 21-35 (1987)

${ }^{4}$ A. W. Dutton, R. B. Roemer, and F. A. Gibbs, "A clinically integrated scanned focused ultrasound hyperthermia," Proceedings of the seventh International Congress on Hyperthermic Oncology, Rome, Italy, 1996 (unpublished), Vol. 1, p. 92.

${ }^{5}$ J. W. Hand, C. C. Vernon, and M. V. Prior, "Early experience of commercial scanned focused ultrasound hyperthermia system," Int. J. Hyperthermia 8, 587-607 (1992).

${ }^{6}$ J. W. Hunt, G. Lockwood, and A. Worthington, "Design of conical transducers to produce strongly focused ultrasound beams for deep heating," Int. J. Hyperthermia 7, 15-28 (1991).

${ }^{7}$ K. Lindsley, P. R. Stauffer, P. Sneed, R. Chin, T. L. Phillips, E. Seppi, E. Shapiro, and S. Henderson, "Heating patterns of the Helios ultrasound hyperthermia system," Int. J. Hyperthermia 9, 675-684 (1993).

${ }^{8}$ W.-L. Lin, J. Y. Yen, Y. Y. Chen, K. S. Cheng, and M. J. Shieh, "Specific absorption rate ratio patterns of cylindrical ultrasound transducers for breast tumors,"' Med. Phys. 25, 1041-1048 (1998).

${ }^{9}$ G. K. Svensson, J. L. Hansen, D. D. Carpini, B. Bornstain, and T. Herman, "SAR and temperature distributions from a spherical focused, segmented ultrasound machine,"' Proceedings of the Sixth International Congress on Hyperthermic Oncology, Tucson, AZ, 1992 (unpublished), p. 335

${ }^{10}$ G. H. Nussbaum, W. L. Straube, M. D. Drag, G. L. Melson, B. Emami, V. Sathiaseelan, E. Seppi, and E. Shapiro, "Potential for localized, adjustable deep heating in soft-tissue environments with a 30-beam ultrasonic hyperthermia system,' Int. J. Hyperthermia 7, 279-299 (1991).

${ }^{11}$ E. Seppi, E. Shapiro, L. Zitelli, S. Henderson, A. Wehlau, G. Wu, and C. Ditmar, "A large aperture ultrasonic array system for hyperthermia of deep-seated tumors," Proceedings of the 1985 IEEE Ultrasonics Symposium, 1985 (unpublished), pp. 942-948.

${ }^{12}$ T. Kirkhorn, L. O. Almquist, H. W. Persson, and N. G. Holmer, "An experimental high-energy therapeutic ultrasound equipment: Design and characterization," Med. Biol. Eng. Comput. 35, 295-299 (1997).

${ }^{13}$ E. S. Ebbini and C. A. Cain, "A spherical-section ultrasound phased array applicator for deep localized hyperthermia,' IEEE Trans. Biomed. Eng. 38, 634-643 (1991)

${ }^{14}$ R. J. McGough, E. S. Ebbini, and C. A. Cain, "Treatment planning for hyperthermia with ultrasound phased arrays," IEEE Trans. Ultrason. Ferroelectr. Freq. Control 43, 1074-1084 (1996).

${ }^{15}$ E. G. Moros, X. Fan, and W. L. Straube, "An investigation of penetration depth control using parallel opposed ultrasound arrays and a scanning reflector,' J. Acoust. Soc. Am. 101, 1734-1741 (1997).

${ }^{16}$ E. G. Moros, W. L. Straube, and R. J. Myerson, "Potential for power deposition conformability using reflected-scanned planar ultrasound," Int. J. Hyperthermia 12, 723-736 (1996).

${ }^{17}$ E. G. Moros, R. B. Roemer, and K. Hynynen, "Simulations of scanned focused ultrasound hyperthermia: The effects of scanning speed and pattern," IEEE Trans. Ultrason. Ferroelectr. Freq. Control 35, 552-560 (1988).

${ }^{18} \mathrm{P}$. P. Lele, "Physical aspects and clinical studies with ultrasound hyperthermia," in Hyperthermia in Cancer Therapy, edited by F. C. Storm (Hall, Boston, 1983), pp. 333-367.

${ }^{19}$ W.-L. Lin, R. B. Roemer, E. G. Moros, and K. Hynynen, "Optimization of temperature distributions in scanned, focused ultrasound hyperthermia," Int. J. Hyperthermia 8, 61-78 (1992).

${ }^{20}$ E. G. Moros, R. B. Roemer, and K. Hynynen, "Pre-focal plane high- 
temperature regions induced by scanning focused ultrasound beams, ' Int. J. Hyperthermia 6, 351-366 (1990).

${ }^{21}$ P. M. Harari, K. Hynynen, R. B. Roemer, D. P. Anhalt, D. S. Shimm, B. Stea, and J. R. Cassady, "Development of scanned focused ultrasound hyperthermia: Clinical response evaluation,' Int. J. Radiat. Oncol., Biol., Phys. 21, 831-840 (1991).

${ }^{22} \mathrm{~K}$. Hynynen, "The threshold for thermally significant cavitation in dog's thigh muscle in vivo," Ultrasound Med. Biol. 17, 157-169 (1991).

${ }^{23}$ W. Swindell, R. B. Roemer, and S. Clegg, "Temperature distributions caused by dynamic scanning of focused ultrasound transducer,' Proceedings of the 1982 IEEE Ultrasonics Symposium (IEEE, New York, 1982), p. 750 .

${ }^{24}$ E. G. Moros, "Simulations of scanned focused ultrasound hyperthermia: The effects of scanning speed, scanning pattern, and multiple tilted transducers," Master's thesis, University of Arizona, 1987.

${ }^{25}$ S. A. Goss, R. L. Johnson, and F. Dunn, "Comprehensive compilation of empirical ultrasonic properties of mammalian tissues,' J. Acoust. Soc. Am. 64, 423-457 (1978).

${ }^{26}$ S. A. Goss, L. A. Frizzell, and F. Dunn, "Ultrasonic absorption and attenuation of high frequency sound in mammalian tissues,' Ultrasound Med. Biol. 5, 181-186 (1979).

${ }^{27}$ S. A. Goss, R. L. Johnson, and F. Dunn, "Compilation of empirical ultrasonic properties of mammalian tissues. II,' J. Acoust. Soc. Am. 68, 93-108 (1980).

${ }^{28} \mathrm{~J}$. C. Bamber and C. R. Hill, "Ultrasonic attenuation and propagation speed in mammalian tissues as a function of temperature," Ultrasound Med. Biol. 5, 149-157 (1979).

${ }^{29}$ H. H. Pennes, "Analysis of tissue and arterial blood temperatures in the resting human forearm,' J. Appl. Phys. 1, 93-122 (1948).

${ }^{30}$ R. K. Jain, "Bio-heat transfer: Mathematical models of thermal sys- tems,' in Hyperthermia Cancer Therapy, edited by F. C. Storm (Hall, Boston, 1983), pp. 9-46.

${ }^{31}$ R. B. Roemer, "Thermal dosimetry," in Thermal Dosimetry and Treatment Planning, edited by M. Gautherie (Springer, Berlin, 1990), pp. 119-214.

${ }^{32}$ J. F. Lehmann, Therapeutic Heat and Cold (Williams and Wilkins, Baltimore, 1982)

${ }^{33}$ NCRP Report No. 113, Exposure Criteria for Medical Diagnostic Ultrasound. I. Criteria based on the Thermal Mechanisms (NCRP, Bethesda, MD, 1992), pp. 52-60.

${ }^{34}$ J. H. Ferziger, Numerical Methods for Engineering Application (Wiley, New York, 1981)

${ }^{35}$ G. D. Simth, Numerical Solution of Partial Differential Equations: Finite Difference Methods (Clarendon, Oxford, 1989).

${ }^{36} \mathrm{H}$. Arkin, X. Xu and K. R. Holmes, "Recent developments in modeling heat transfer in blood perfusion tissues,' IEEE Trans. Biomed. Eng. 41, 97-107 (1994).

${ }^{37}$ J. C. Chato, "Fundamentals of bio-heat transfer,' in Thermal Dosimetry and Treatment Planning, edited by M. Gautherie (Springer, Berlin, 1990), pp. 1-56.

${ }^{38}$ W.-L. Lin, J. Y. Yen, Y. Y. Chen, K. W. Jin, and M. J. Shieh, "Relationship between acoustic aperture size and tumor conditions for external ultrasound hyperthermia,', Med. Phys. 26, 818-824 (1999).

${ }^{39}$ K. Hynynen, D. J. Watmough, and J. R. Mallard, “'Design of ultrasonic transducers for local hyperthermia,' Ultrasound Med. Biol. 7, 397-402 (1981).

${ }^{40}$ R. B. Roemer, "'Optimal power deposition in hyperthermia. I. The treatment goal, the ideal temperature distribution, the role of large blood vessels,' Int. J. Hyperthermia 7, 317-342 (1991). 